\title{
Thermodynamic evidence for a nematic phase transition at the onset of the pseudogap
} \section{in $\mathrm{YBa}_{2} \mathrm{Cu}_{3} \mathrm{O}_{y}$}

\author{
Y. Sato', S. Kasahara1, H. Murayama1, Y. Kasahara', E.-G. Moon², T. Nishizaki ${ }^{3}$, T. Loew ${ }^{4}$, J. Porras ${ }^{4}$, \\ B. Keimer ${ }^{4}$, T. Shibauchi ${ }^{5}$ and Y. Matsuda ${ }^{1 \star}$
}

\begin{abstract}
A long-standing controversial issue in the quest to understand the superconductivity in cuprates is the nature of the enigmatic pseudogap region of the phase diagram'. Especially important is whether the pseudogap state is a distinct thermodynamic phase characterized by broken symmetries below the onset temperature $T^{*}$. Here we report torque-magnetometry measurements of anisotropic susceptibility within the $a b$ planes in orthorhombic $\mathrm{YBa}_{2} \mathrm{Cu}_{3} \mathrm{O}_{y}$ with exceptionally high precision. The in-plane anisotropy displays a significant increase with a distinct kink at the pseudogap onset temperature $T^{*}$, showing a remarkable scaling behaviour with respect to $T / T^{*}$ in a wide doping range. Our systematic analysis reveals that the rotational symmetry breaking sets in at $T^{*}$ in the limit where the effect of orthorhombicity is eliminated. These results provide thermodynamic evidence that the pseudogap onset is associated with a second-order nematic phase transition, which differs from the recently reported charge-density-wave transition that accompanies translational symmetry breaking ${ }^{2-10}$.
\end{abstract}

The pseudogap state harbours anomalous electronic states such as Fermi arcs, charge density waves (CDW), and $d$-wave superconductivity $^{1}$. Electronic nematicity, a four-fold $\left(C_{4}\right)$ rotational symmetry breaking, has emerged as a key feature inside the pseudogap regime $e^{11-14}$, but the presence or absence of a nematic phase transition and its relationship to the pseudogap remain unresolved. Nematicity has been widely discussed in cuprates, and one of its mechanism is the onset of a stripe-type CDW order parameter which generally breaks rotation symmetry as well as translation symmetry with a nonzero wavenumber $\mathbf{Q} \neq 0$ (refs 2-10,15-18). In $\mathrm{Bi}_{2} \mathrm{Sr}_{2} \mathrm{CaCu}_{2} \mathrm{O}_{8+\delta}$ (BSCCO), scanning tunnelling microscopy experiments at low temperatures report an electronic state, consisting of short-range CDW of unidirectional (one-dimensional, 1D) type with a period of $\sim 4 a_{0}$, where $a_{0}$ is the $\mathrm{Cu}-\mathrm{O}-\mathrm{Cu}$ distance ${ }^{19,20}$. This nano-stripe structure persists even well above the superconducting transition temperature $T_{c}$ (ref. 21). In $\mathrm{YBa}_{2} \mathrm{Cu}_{3} \mathrm{O}_{y}$ (YBCO), the short-range CDW order forms a dome-shaped boundary inside the pseudogap regime ${ }^{6,7}$. Resonant X-ray scattering (RXS) experiments in YBCO report that the CDW is of unidirectional type with a periodicity of $\sim 3 a_{0}$ (ref. 4 ). In both BSCCO and YBCO, the CDW forms domains with a size of $\sim 3 \mathrm{~nm}$ in zero field, inside which the $C_{4}$ symmetry of the unit cell is strongly broken. In contrast to such CDW orders, the nematicity may also be caused by an instability without breaking translational symmetry, characterized by $\mathbf{Q}=0$.
The measurement of the magnetic torque has a very high sensitivity for detecting magnetic anisotropy. The torque $\tau=$ $\mu_{0} V \mathbf{M} \times \mathbf{H}$ is a thermodynamic quantity, a differential of the free energy with respect to angular displacement. Here $\mu_{0}$ is the permeability of vacuum, $V$ is the sample volume, and $\mathbf{M}$ is the magnetization induced by the external magnetic field $\mathbf{H}$. When $\mathbf{H}$ is rotated within the $a b$ plane, $\tau$ is a periodic function of double the azimuthal angle $\phi$ measured from the $a$ axis (Fig. 1a):

$$
\tau_{2 \phi}=\frac{1}{2} \mu_{0} H^{2}\left[\left(\chi_{a a}-\chi_{b b}\right) \sin 2 \phi-2 \chi_{a b} \cos 2 \phi\right]
$$

where the susceptibility tensor $\chi_{i j}$ is given by $M_{i}=\Sigma_{j} \chi_{i j} H_{j}$. In a system with tetragonal symmetry, $\tau_{2 \phi}$ should be zero. When $C_{4}$ symmetry is broken, a nonzero $\tau_{2 \phi}$ appears as a result of $\chi_{a a} \neq \chi_{b b}$ and/or $\chi_{a b} \neq 0$, depending on the orthorhombicity direction.

Figure $1 \mathrm{~b}$ displays typical curves of magnetic torque measured as a function of $\phi$. All the curves are perfectly sinusoidal, but deviation occurs only near $T_{c}$ due to diamagnetic susceptibility originating from superconducting fluctuations (see Methods and Supplementary Information). In the temperature range shown here, the oscillations are proportional to $\sin 2 \phi$ with a positive sign, indicating $\chi_{a a}>\chi_{b b}$ and $\chi_{a b}=0$ (Fig. 1c). Figure 2a-c depicts the amplitude of the in-plane anisotropy of the susceptibility, $\Delta \chi_{\|} \equiv \chi_{a a}-\chi_{b b}$, at $\mu_{0} H=7 \mathrm{~T}$ for underdoped YBCO with $T_{\mathrm{c}}=60,70$ and $90 \mathrm{~K}$ (hole concentration $p \approx 0.11,0.13$, and 0.15 ), respectively. In all the crystals, as the temperature is lowered, $\Delta \chi_{\|}$gradually decreases, then increases rapidly after exhibiting a distinct kink at $T=T_{\tau}$. Since the average of uniform susceptibilities $\chi_{a a}$ and $\chi_{b b}$ is also temperature dependent, we introduce a dimensionless order parameter $\eta \equiv\left(\chi_{a a}-\chi_{b b}\right) /\left(\chi_{a a}+\chi_{b b}\right)$, a diagonal component of a nematic traceless symmetric tensor in two spatial dimensions, to discuss the nematicity properly (see Fig. $2 \mathrm{~d}-\mathrm{f}$, and Methods). Above $T_{\tau}, \eta(T)$ is nearly independent of temperature, indicating that the uniform susceptibility causes a weak temperature dependence of $\Delta \chi_{\|}$above $T_{\tau}$. Below $T_{\tau}, \eta(T)$ increases with a slightly concave curvature.

Figure 3 displays the temperature-doping phase diagram of YBCO. Obviously $T_{\tau}$ coincides well with the pseudogap temperature $T^{*}$ determined by various other probes. In what follows, we identify $T_{\tau}$ as the pseudogap onset temperature $T^{*}$ (that is, $T_{\tau}=T^{*}$ ). The kink anomaly in the temperature dependence of $\Delta \chi_{\|}$is usually an indication of a second-order phase transition. However, the $\mathrm{C}_{4}$ symmetry is already broken due to the orthorhombic crystal structure with $1 \mathrm{D} \mathrm{CuO}$ chains in $\mathrm{YBCO}$, and thus no further

${ }^{1}$ Department of Physics, Kyoto University, Kyoto 606-8502, Japan. ${ }^{2}$ Department of Physics, Korea Advanced Institute of Science and Technology, Daejeon 305-701, Korea. ${ }^{3}$ Department of Electrical Engineering, Kyushu Sangyo University, Fukuoka 813-8503, Japan. ${ }^{4}$ Max Planck Institute for Solid State Research, Heisenbergstraße 1, D-70569 Stuttgart, Germany. ${ }^{5}$ Department of Advanced Materials Science, University of Tokyo, Chiba 277-8561, Japan. *e-mail: matsuda@scphys.kyoto-u.ac.jp 

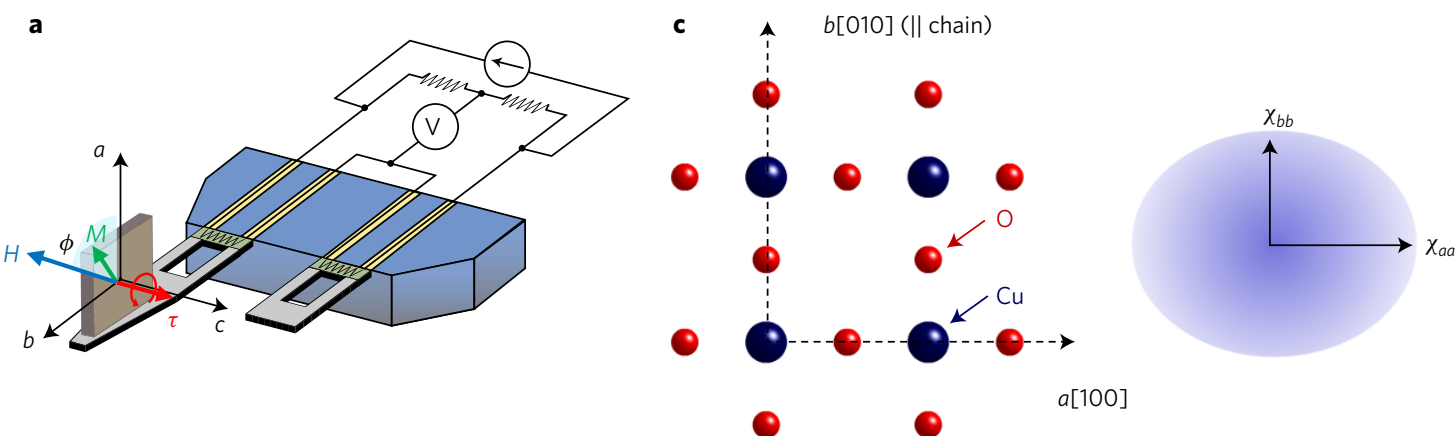

b

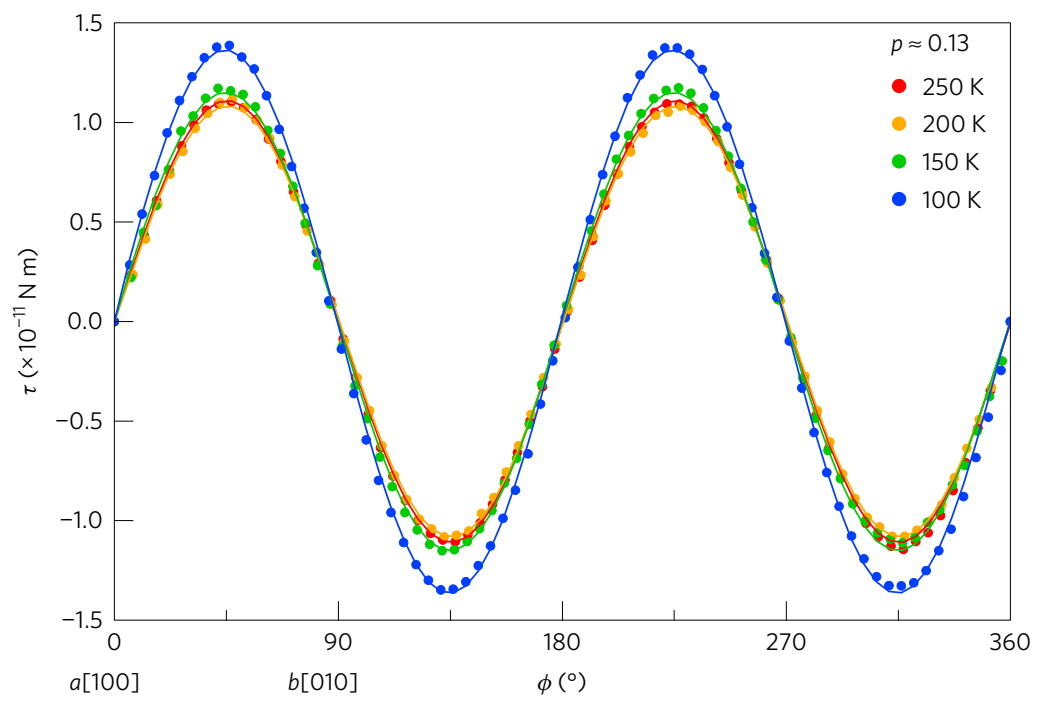

Figure 1 | In-plane torque magnetometry in YBCO single crystals. a, Schematic representation of the experimental configuration for torque measurements under an in-plane field rotation. An untwinned single-crystalline sample of YBCO is mounted on the piezo-resistive lever, which forms an electrical bridge circuit with the neighbouring reference lever. $\mathbf{b}$, Typical curves of magnetic torque $\tau$ in YBCO $(p \sim 0.13)$ as a function of the azimuthal angle $\phi$ from the $a$ axis. A field of $\mu_{0} \mathrm{H}=7 \mathrm{~T}$ is applied within the $a b$ plane with a misalignment of less than $0.1^{\circ}$. c, Schematic view of the $\mathrm{CuO}_{2}$ plane. Because of the orthorhombic crystal structure stabilized by $\mathrm{CuO}$ chains ( $\| b$ axis) lying between the $\mathrm{CuO}_{2}$ bilayers, $\chi_{a a}>\chi_{b b}$.

rotational symmetry breaking is expected. Indeed, $\eta(T)$ is finite even above $T^{*}$ for all the samples (Fig. 2d-f), confirming that the orthorhombic structure generally leads to anisotropic magnetic susceptibility. We note that the magnitude of $\eta\left(T^{*}\right)$ increases with hole doping, which may be explained by the increased crystal orthorhombicity through the oxidation of $\mathrm{CuO}$ chains with doping.

Disentangling the intrinsic electronic nematicity in the $\mathrm{CuO}_{2}$ planes from extrinsic effects due to crystal orthorhombicity has been a vexing issue, particularly in YBCO. It should be stressed that the doping dependence of $\eta(T)$ enables us to examine the nematicity in the limit where the effect of orthorhombicity is removed. Since $\eta(T)$ is independent of temperature above $T^{*}, \eta\left(T^{*}\right)$ represents the background anisotropy stemming from the crystal orthorhombicity. To eliminate this background contribution, we introduce the excess nematicity below $T^{*}, \Delta \eta(T) \equiv \eta(T)-\eta\left(T^{*}\right)$, and plot it as a function of background anisotropy $\eta\left(T^{*}\right)$ (Fig. 4a). As shown by the solid lines, $\Delta \eta(T)$ is nearly proportional to $\eta\left(T^{*}\right)$. Obviously the solid lines have finite intercepts, indicating that even when the crystal orthorhombicity is removed, nematicity remains finite below $T^{*}$; that is, spontaneous $C_{4}$ rotational symmetry breaking in the pseudogap state. This result, along with the kink anomaly of the inplane torque (Fig. 2a-c), provides evidence for a second-order phase transition at $T^{*}$ in the $\mathrm{CuO}_{2}$ planes of YBCO.

The second-order nematic phase transition at $T^{*}$ is further supported by the scaling property of the nematicity for crystals with various hole concentrations. Although $T^{*}$ and $\eta\left(T^{*}\right)$ both have strong doping dependence, the excess anisotropy $\Delta \eta(T)$ exhibits a good scaling behaviour when plotted as a function of $T / T^{*}$. Figure $4 \mathrm{~b}$ depicts $\Delta \eta(T)$ normalized by the value at $T=0.7 T^{*}$ versus $T / T^{*}$. All the curves collapse into a single curve in a wide temperature range. Moreover, the data in the limit of no background anisotropy (Fig. 4a) lie on the same curve. It is well known that genuine second-order phase transitions do not occur in the presence of an external symmetry-breaking field and the kink-type temperature dependence of order parameters will be smeared out. However, if the external field is small enough, the kink behaviours are modified only slightly near the transition points, and scaling properties should prevail. In the present case, the crystal orthorhombicity in YBCO is the external symmetry-breaking field. At the same time, orthorhombic distortion would be helpful in preventing the formation of microscopic domains with orthogonal nematic directions, and thus important for the two-fold nematic signals to be observed in the bulk measurements ${ }^{22}$. The data collapse onto the universal curve in Fig. $4 \mathrm{~b}$ indicates that the influence of the background anisotropy on the nematic order parameter is small except in the vicinity of $T^{*}$. This supports that the crystal orthorhombicity is a small perturbation on the second-order transition and reinforces our analysis using background subtraction. The characteristic super-linear temperature dependence of $\Delta \eta(T)$ appears at the onset of the nematicity. If one interprets the temperature dependence with the critical exponent $\beta, \Delta \eta(T) \propto\left(T^{*}-T\right)^{\beta}$, then the critical exponent shows large deviations from all known results of two-dimensional nematic transitions; for example, those of the mean-field $(\beta=1 / 2)$ and the 2D Ising model $(\beta=1 / 8)$. Although the super-linear dependence 
a

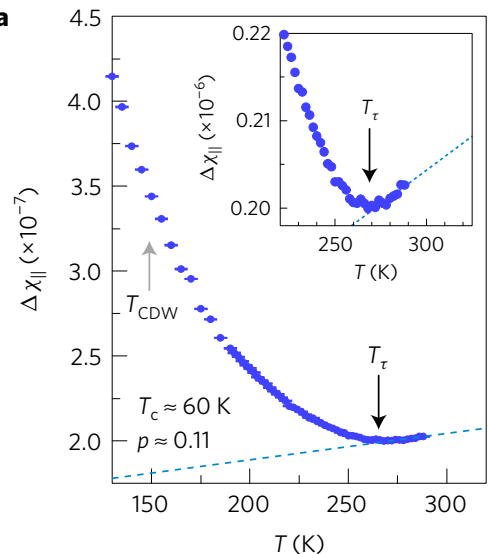

d

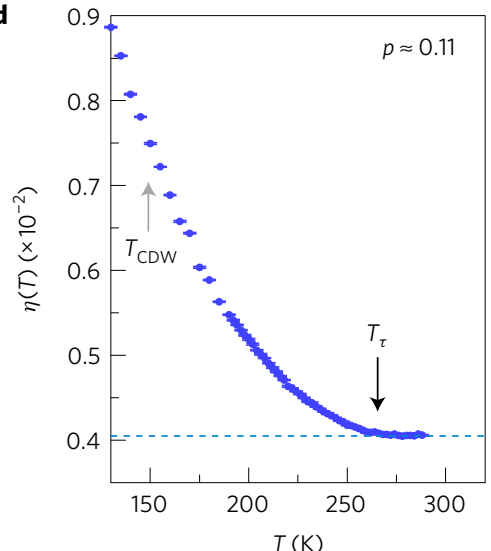

b

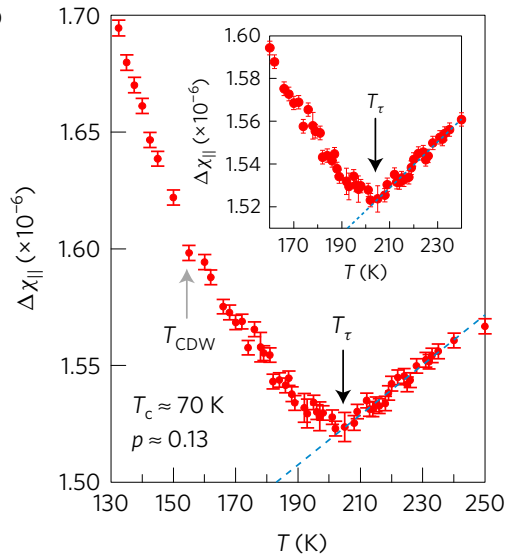

e

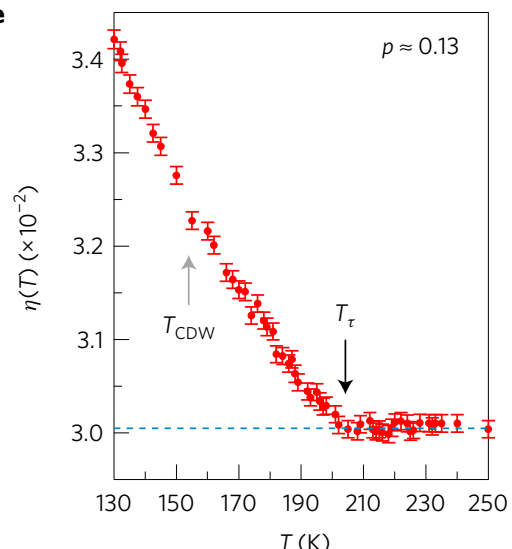

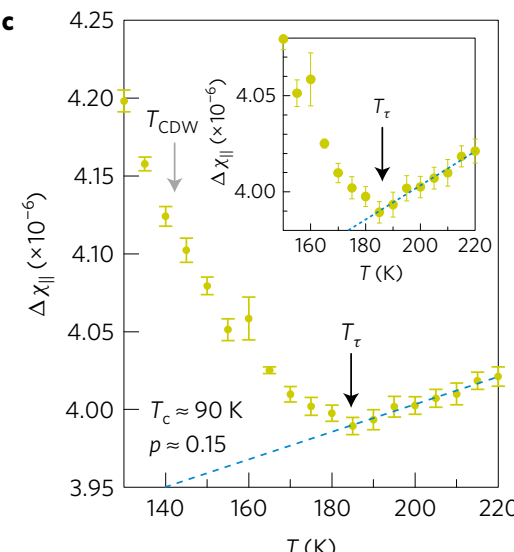

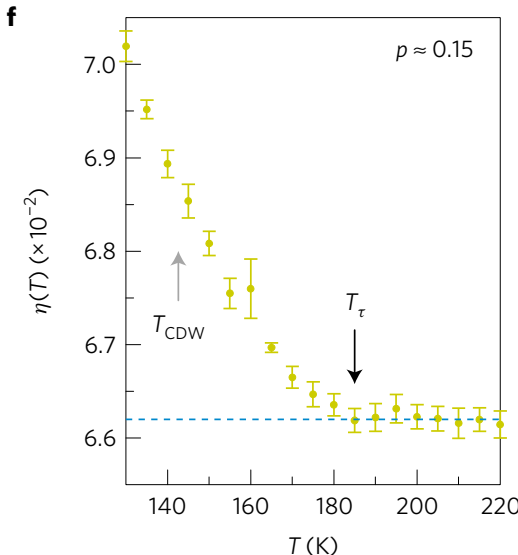

Figure $\mathbf{2}$ | In-plane anisotropy of the magnetic susceptibility for underdoped YBCO with different hole doping levels. a-c, Temperature dependence of the in-plane anisotropy of the magnetic susceptibility, $\Delta \chi_{\|}=\chi_{a a}-\chi_{b b}$, determined from the torque curves for underdoped YBCO with hole concentration $p \approx 0.11,0.13$ and 0.15 , respectively. For all crystals, $\Delta \chi \|$ decreases gradually down to $T_{\tau}$, then increases rapidly below $T_{\tau}$ after exhibiting a kink at $T_{\tau}$. d-f, Temperature dependence of the order parameter $\eta \equiv\left(\chi_{a a}-\chi_{b b}\right) /\left(\chi_{a a}+\chi_{b b}\right)$ for $p \approx 0.11,0.13$ and 0.15 (see Methods for detailed procedure to deduce $\eta$ ), respectively. Above $T_{\tau}, \eta(T)$ is independent of temperature, whereas it shows steep increases below $T_{\tau}$. In sharp contrast to the anomaly at $T_{\tau}$, no discernible anomaly is observed at the CDW transition temperature $T_{\mathrm{CDW}}$. The background anisotropy due to the crystal orthorhombicity, $\eta\left(T_{\tau}\right)$, increases rapidly with $p$ (see also Methods).

may be due to some nontrivial temperature dependence of the nematic domain size, the unusual exponent may be associated with a novel transition in a very different universality class, which calls for further theoretical investigation, including scenarios of composite order parameters with randomness and doped spin liquids $s^{23,24}$.

Although the second-order phase transition at $T^{*}$ has been suggested by several experiments, it is far from settled. Resonant ultrasound spectroscopy experiments report a critical slowing down behaviour in the ultrasound absorption as $T^{*}$ is approached ${ }^{25}$, but a different interpretation without critical phenomena has been proposed $^{26}$. The polarized neutron scattering experiments report time reversal symmetry breaking (TRSB) with the appearance of a magnetic moment at $T^{*}$, which has been interpreted as circulating current loops within the $\mathrm{CuO}_{2}$ unit cell ${ }^{27,28}$. However, polar Kerr effect measurements report that the TRSB temperature is significantly different from $T^{*}$ (ref. 29). Enhancement of the in-plane anisotropy of the Nernst coefficient at $T^{*}$ has been reported ${ }^{22}$, but more recent results have shown that such an enhancement is much more pronounced below $T_{\mathrm{CDW}}$ rather than $T^{*}$ (ref. 30). These results are in sharp contrast to our torque experiments, in which no discernible anomalies are observed at $T_{\mathrm{CDW}}$. Recent RXS experiments report the appearance of orthogonal CDW domains with $\mathbf{Q}_{1} \approx(1 / 3,0, L)$ and $\mathbf{Q}_{2} \approx(0,1 / 3, L)$ (ref. 4$)$. Our results suggest that the effective cancellation of the nematicity occurs due to nearly equal numbers of these CDW domains. We also point out that no anomaly in $\eta(T)$ is expected at $T_{\mathrm{CDW}}$ when a CDW of bidirectional type (chequerboard $)^{5,10,16}$, which preserves rotational symmetry, is formed.

Our results clearly demonstrate that the pseudogap state is a thermodynamic phase with nematic character. The phase diagram of hole-doped cuprate superconductors (Fig. 3) then include at least four different ordered phases; antiferromagnetic, superconducting, CDW, and pseudogap phases, which are characterized by broken time, gauge, translational, and rotational symmetries, respectively. The question as to whether the nematicity is the primary cause or a secondary instability of the transition remains open. Further work is needed to clarify the relationship between the present results, the $\mathbf{Q}=0$ magnetic order ${ }^{27}$, and the spontaneous onset of inplane anisotropies observed in the low-energy spin excitations ${ }^{14}$, the electrical resistivity ${ }^{13}$, and the Nernst effect ${ }^{22}$.

Angle-resolved photoemission spectroscopy (ARPES) experiments in BSCCO and related compounds revealed Fermi arc formation, where the Fermi surface partially disappears in the pseudogap state $^{31}$. Yet, important questions still remain-for instance, the link between the nematic transition and Fermi arc formation and the interplay between the pseudogap and CDW. Whether a quantum critical point (QCP) is present inside the superconducting dome has been a hotly debated issue in cuprates ${ }^{1,25,26}$. The presence of a QCP has been suggested by several measurements around $p \approx 0.19$ in $\mathrm{YBCO}^{32,33}$ and at a slightly higher doping in $\mathrm{BSCCO}^{28,31,34}$. The identification of the pseudogap temperature as the critical temperature of a second-order nematic transition favours the QCP scenario; that 


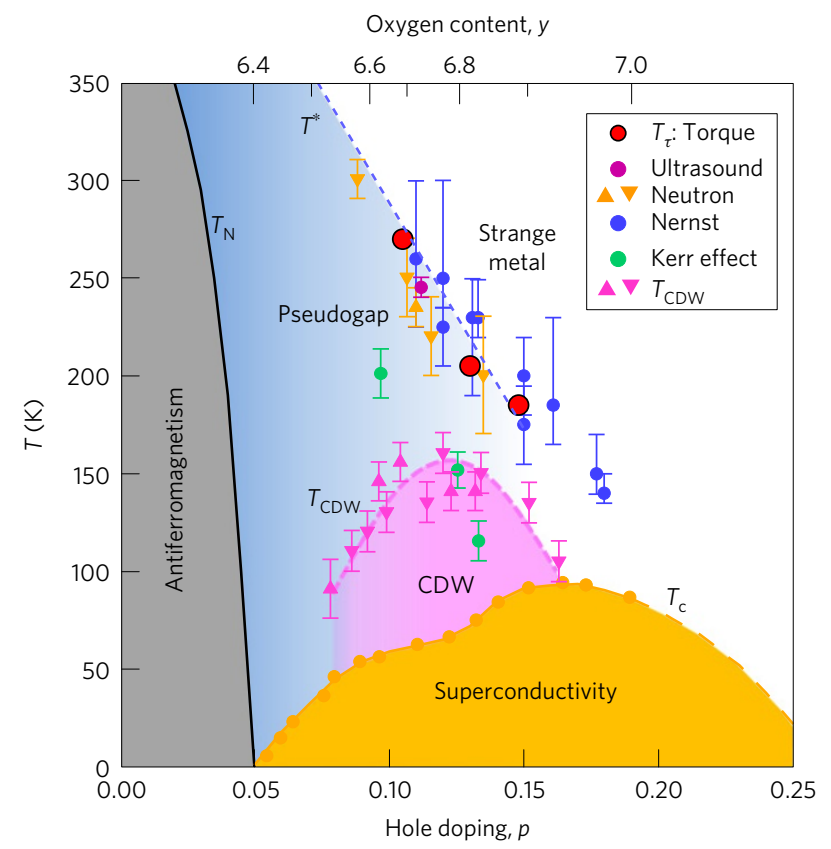

Figure 3 | Temperature-doping phase diagram of YBCO. The phase diagram contains at least four different ordered phases, including antiferromagnetism (grey), superconductivity (yellow), CDW (pink) and pseudogap (blue) regimes. The pseudogap line (dashed line) at $T^{*}$ marks the boundary between the strange metal and even more anomalous regimes. Red circles represent the second-order nematic transition temperature $T_{\tau}$ determined by the present in-plane torque magnetometry. For comparison, the pseudogap temperatures determined by other probes are also plotted. Purple circles, orange triangles and blue circles are $T^{*}$ reported by ultrasound spectroscopy ${ }^{25}$, polarized neutron scattering ${ }^{27}$, and Nernst coefficients ${ }^{22}$, respectively. Magenta triangles represent the formation temperature of the short-range CDW, $T_{\mathrm{CDW}}$, reported by resonant $X$-ray measurements ${ }^{6,7}$. Green circles are the temperature below which the time reversal symmetry is broken, reported by the polar Kerr effect ${ }^{29}$.

is, the extension of the pseudogap temperature to $T \rightarrow 0$ suggests a nematic QCP. The second-order nature of the phase transition line, in general, implies the presence of critical fluctuations near the transition line, and in an extended regime around the QCP one may expect significant quantum critical fluctuations. Hence it is tempting to consider that the nematic quantum fluctuations influence the superconductivity as well as the strange metallic behaviour in the normal state of cuprates.

\section{Methods}

Methods, including statements of data availability and any associated accession codes and references, are available in the online version of this paper.

Received 28 November 2016; accepted 14 June 2017; published online 24 July 2017

\section{References}

1. Keimer, B., Kivelson, S. A., Norman, M. R., Uchida, S. \& Zaanen, J. From quantum matter to high-temperature superconductivity in copper oxides. Nature 518, 179-186 (2015).

2. Ghiringhelli, G. et al. Long-range incommensurate charge fluctuations in (Y,Nd) $\mathrm{Ba}_{2} \mathrm{Cu}_{3} \mathrm{O}_{6+x}$. Science 337, 821-825 (2012).

3. Chang, J. et al. Direct observation of competition between superconductivity and charge density wave order in $\mathrm{YBa}_{2} \mathrm{Cu}_{3} \mathrm{O}_{6.67}$. Nat. Phys. 8, 871-876 (2012).

4. Comin, R. et al. Broken translational and rotational symmetry via charge stripe order in underdoped $\mathrm{YBa}_{2} \mathrm{Cu}_{3} \mathrm{O}_{6+y}$. Science 347, 1335-1339 (2015).
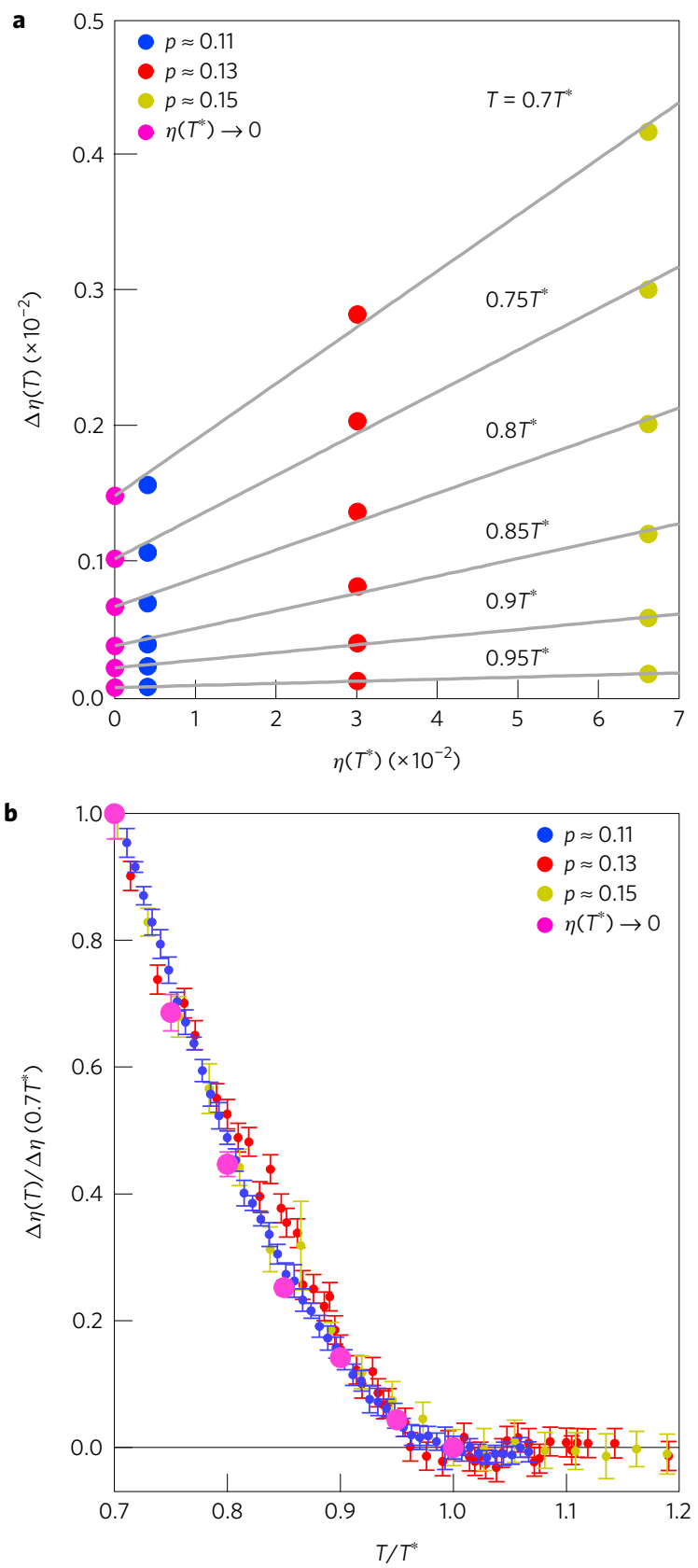

Figure 4 | Induced nematicity and the scaling behaviour. a, The excess anisotropy below $T^{*}, \Delta \eta(T) \equiv \eta(T)-\eta\left(T^{*}\right)$, of YBCO with different hole concentrations $p$, plotted as a function of the background anisotropy $\eta\left(T^{*}\right)$ at different values of $T / T^{*}$. The solid lines represent the linear fit for $\Delta \eta(T)$ at each value of $T / T^{*}$. The magenta symbols show the induced nematicity in the limit of $\eta\left(T^{*}\right) \rightarrow 0$. b. The excess anisotropy $\Delta \eta(T)$ normalized by the value at $T / T^{*}=0.7$ plotted as a function of $T / T^{*}$. All the data collapse into a universal curve, indicating a scaling relation.

5. Forgan, E. M. et al. The microscopic structure of charge density waves in underdoped $\mathrm{YBa}_{2} \mathrm{Cu}_{3} \mathrm{O}_{6.54}$ revealed by $\mathrm{X}$-ray diffraction. Nat. Commun. 6, 10064 (2015).

6. Hücker, M. et al. Competing charge, spin, and superconducting orders in underdoped $\mathrm{YBa}_{2} \mathrm{Cu}_{3} \mathrm{O}_{y}$. Phys. Rev. B 90, 054514 (2014).

7. Blanco-Canosa, S. et al. Resonant X-ray scattering study of charge-density wave correlations in $\mathrm{YBa}_{2} \mathrm{Cu}_{3} \mathrm{O}_{6+x}$. Phys. Rev. B 90, 054513 (2014).

8. Wu, T. et al. Incipient charge order observed by NMR in the normal state of $\mathrm{YBa}_{2} \mathrm{Cu}_{3} \mathrm{O}_{y}$. Nat. Commun. 6, 6438 (2015).

9. Jang, H. et al. Ideal charge density wave order in the high-field state of superconducting YBCO. Proc. Natl Acad. Sci. USA 113, 14645-14650 (2016). 
10. Sebastian, S. E. \& Proust, C. Quantum oscillations in hole-doped cuprates. Annu. Rev. Condens. Mater. Phys. 6, 411-430 (2015).

11. Kivelson, S. A., Fradkin, E. \& Emery, V. J. Electronic liquid-crystal phases of a doped Mott insulator. Nature 393, 550-553 (1998).

12. Vojta, M. Lattice symmetry breaking in cuprate superconductors: stripes, nematics, and superconductivity. Adv. Phys. 58, 699-820 (2009).

13. Ando, Y., Segawa, K., Komiya, S. \& Lavrov, A. N. Electrical resistivity anisotropy from self-organized one dimensionality in high-temperature superconductors. Phys. Rev. Lett. 88, 137005 (2002).

14. Hinkov, V. et al. Electronic liquid crystal state in the high-temperature superconductor $\mathrm{YBa}_{2} \mathrm{Cu}_{3} \mathrm{O}_{6.45}$. Science 319, 597-600 (2008).

15. Scalapino, D. J. \& White, S. R. Stripe structures in the $t-t^{\prime}-J$ model. Physica C 481, 146-152 (2012).

16. Wang, Y. \& Chubukov, A. Charge-density-wave order with momentum $(2 Q, 0)$ and $(0,2 Q)$ within the spin-fermion model: continuous and discrete symmetry breaking, preemptive composite order, and relation to pseudogap in hole-doped cuprates. Phys. Rev. B 90, 035149 (2014).

17. Yamakawa, Y. \& Kontani, H. Spin-fluctuation-driven nematic charge-density wave in cuprate superconductors: impact of Aslamazov-Larkin vertex corrections. Phys. Rev. Lett. 114, 257001 (2015).

18. Schütt, M. \& Fernandes, R. M. Antagonistic in-plane resistivity anisotropies from competing fluctuations in underdoped cuprates. Phys. Rev. Lett. 115, 027005 (2015).

19. Kohsaka, Y. et al. An intrinsic bond-centered electronic glass with unidirectional domains in underdoped cuprates. Science $\mathbf{3 1 5}$, 1380-1385 (2007).

20. Lawler, M. J. et al. Intra-unit-cell electronic nematicity of the high- $T_{c}$ copper-oxide pseudogap states. Nature 466, 347-351 (2010).

21. Parker, C. V. et al. Fluctuating stripes at the onset of the pseudogap in the high- $T_{\mathrm{c}}$ superconductor $\mathrm{Bi}_{2} \mathrm{Sr}_{2} \mathrm{CaCu}_{2} \mathrm{O}_{8+x}$. Nature 468, 677-680 (2010).

22. Daou, R. et al. Broken rotational symmetry in the pseudogap phase of a high- $T_{\mathrm{c}}$ superconductor. Nature 463, 519-522 (2010).

23. Nie, L., Tarjus, G. \& Kivelson, S. Quenched disorder and vestigial nematicity in the pseudogap regime of the cuprates. Proc. Natl Acad. Sci. USA 111, 7980-7985 (2014).

24. Patel, A., Chowdhury, D., Allais, A. \& Sachdev, S. Confinement transition to density wave order in metallic doped spin liquids. Phys. Rev. B 93, 165139 (2016).

25. Shekhter, A. et al. Bounding the pseudogap with a line of phase transitions in $\mathrm{YBa}_{2} \mathrm{Cu}_{3} \mathrm{O}_{6+\delta}$. Nature 498, 75-77 (2013).

26. Cooper, J. R., Loram, J. W., Kokanović, I., Storey, J. G. \& Tallon, J. L. Pseudogap in $\mathrm{YBa}_{2} \mathrm{Cu}_{3} \mathrm{O}_{6+\delta}$ is not bounded by a line of phase transitions: thermodynamic evidence. Phys. Rev. B 89, 201104(R) (2014).

27. Fauqué, B. et al. Magnetic order in the pseudogap phase of high- $T_{\mathrm{c}}$ superconductors. Phys. Rev. Lett. 96, 197001 (2006).
28. Mangin-Thro, L. et al. Characterization of the intra-unit-cell magnetic order in $\mathrm{Bi}_{2} \mathrm{Sr}_{2} \mathrm{CaCu}_{2} \mathrm{O}_{8+\delta}$. Phys. Rev. B 89, 094523 (2014).

29. Xia, J. et al. Polar Kerr-effect measurements of the high-temperature $\mathrm{YBa}_{2} \mathrm{Cu}_{3} \mathrm{O}_{6+x}$ superconductor: evidence for broken symmetry near the pseudogap temperature. Phys. Rev. Lett. 100, 127002 (2008).

30. Cyr-Choiniére, O. et al. Two types of nematicity in the phase diagram of the cuprate superconductor $\mathrm{YBa}_{2} \mathrm{Cu}_{3} \mathrm{O}_{y}$. Phys. Rev. B 92, 224502 (2015).

31. Hashimoto, M., Vishik, I. M., He, R.-H., Devereaux, T. P. \& Shen, Z.-X. Energy gaps in high-transition-temperature cuprate superconductors. Nat. Phys. 10, 483-495 (2014)

32. Tallon, J. L. \& Loram, J. W. The doping dependence of $T^{*}$ - what is the real high- $T_{\mathrm{c}}$ phase diagram? Physica C 349, 53-68 (2001)

33. Badoux, S. et al. Change of carrier density at the pseudogap critical point of a cuprate superconductor. Nature 531, 210-214 (2016).

34. Benhabib, S. et al. Collapse of the normal-state pseudogap at a Lifshitz transition in the $\mathrm{Bi}_{2} \mathrm{Sr}_{2} \mathrm{CaCu}_{2} \mathrm{O}_{8+\delta}$ cuprate superconductor. Phys. Rev. Lett. 114, $147001(2015)$

\section{Acknowledgements}

We thank A. Carrington, R. M. Fernandes, T. Hanaguri, N. Harrison, S. M. Hayden, M.-H. Julien, S. Kivelson, H. Kontani, C. Putzke, T. M. Rice, S. Sachdev, L. Taillefer, T. Tohyama, H. Yamase and J. Zaanen for fruitful discussions, and M. Ishikawa and H. Yamochi for experimental support. This work was supported by Grants-in-Aid for Scientific Research (KAKENHI) (Nos. 25220710, 15H02106, 15H03688, 16K05460, 16K13837) and on Innovative Areas 'Topological Material Science' (No. 15H05852) from Japan Society for the Promotion of Science (JSPS). The characterization of YBCO single crystals was partly performed at Advanced Instruments Center at Kyushu Sangyo University. E.-G.M. acknowledges the financial supports from the POSCO Science Fellowship of POSCO TJ Park Foundation and NRF of Korea under Grant No. 2017R1C1B2009176.

\section{Author contributions}

T.N., T.L., J.P. and B.K. prepared the high-quality single-crystalline samples. Y.S., H.M. and S.K. performed the magnetic torque measurements. Y.S., S.K., E.-G.M. and Y.M. analysed the data. S.K., E.-G.M., Y.K., T.S., B.K. and Y.M. discussed and interpreted the results and prepared the manuscript.

\section{Additional information}

Supplementary information is available in the online version of the paper. Reprints and permissions information is available online at www.nature.com/reprints. Publisher's note: Springer Nature remains neutral with regard to jurisdictional claims in published maps and institutional affiliations. Correspondence and requests for materials should be addressed to Y.M.

\section{Competing financial interests}

The authors declare no competing financial interests. 


\section{Methods}

Materials. High-quality single crystals of YBCO were grown by the flux method ${ }^{7,35}$. The oxygen concentration was controlled by annealing the crystals at high temperatures under an oxygen or a nitrogen flow atmosphere ${ }^{7,36}$. The hole doping levels were determined from $c$ axis data ${ }^{37}$. For $p \approx 0.11,0.13$ and 0.15 , we used naturally untwinned single crystals which were carefully selected under a polarized microscope. The crystal of $p \approx 0.10$ was mechanically detwinned by heating under uniaxial stress ${ }^{7,38}$. The superconducting transition temperature $T_{\mathrm{c}}$ was characterized by the magnetization measurements. The crystals exhibit sharp superconducting transitions, with the value of $T_{\mathrm{c}}$ determined as the midpoint at 58 , 60,70 and $90 \mathrm{~K}$ for $p \approx 0.10,0.11,0.13$ and 0.15 , respectively ${ }^{7,36}$. A first-order vortex-lattice melting transition, which can be seen only in clean and homogeneous single crystals, is clearly observed in the crystals prepared by the same method ${ }^{39-41}$, indicating the high quality of our crystals. For each crystal, the directions of the $a$ and $b$ axes were determined by $\mathrm{X}$-ray diffraction.

Torque magnetometry. Magnetic torque is measured by the piezo-resistive micro-cantilever technique, which is a very sensitive probe of magnetic anisotropy $\mathrm{y}^{42-45}$. In this method, an isotropic Curie contribution from impurity spins is cancelled out ${ }^{44}$. Carefully selected single crystals with typical dimensions of $250 \times 250 \times 50 \mu^{3}$ are used in the torque measurements. The in-plane and out-of-plane anisotropies of the magnetic susceptibilities can be measured depending on the geometry of the sample, which is mounted on the lever (see Fig. 1a and Supplementary Fig. 1a).

Supplementary Fig. $1 \mathrm{~b}$ demonstrates typical magnetic torque $\tau$ in underdoped $\operatorname{YBCO}(p \approx 0.13)$ when a field $\mathbf{H}$ is rotated within a plane including the $c$ axis (out-of-plane torque magnetometry). Here, $\theta$ is the polar angle from the $c$ axis. We note that all of the single crystals used in the present study exhibit a purely paramagnetic response with negligibly small hysteresis components. The $\tau(\theta)$ curves are perfectly sinusoidal, and are fitted well by

$$
\tau_{2 \theta}(\theta, T, H)=\frac{1}{2} \mu_{0} H^{2} V \Delta \chi_{\perp} \sin 2 \theta
$$

where $V$ is the sample volume, $\mu_{0}$ is the permeability of vacuum and $\Delta \chi_{\perp}=\chi_{c c}-\chi_{\|}$is the difference between the $c$ axis and the in-plane susceptibilities, which yields $\pi$ periodic oscillations with respect to $\theta$ rotation.

From the amplitude of the $\tau(\theta)$ curves, we obtain the temperature dependence of $\Delta \chi_{\perp}$ in YBCO with the hole concentration $p \approx 0.11,0.13$ and 0.15 (Supplementary Fig. 2a,b and c, respectively). At high temperatures, the magnitude of $\Delta \chi_{\perp}$ changes nearly linearly with temperature. Upon cooling, the data deviate downward from the linear temperature behaviour below $T^{*}$, which represents the pseudogap formation. This is because the decrease in the density of states results in suppression of the $c$-axis susceptibility as previously reported, for example, in $\mathrm{Bi}_{2} \mathrm{Sr}_{2} \mathrm{CaCu}_{2} \mathrm{O}_{8+\delta}$ (refs 46,47). We note that $T^{*}$ determined by the out-of-plane torque magnetometry coincides well with the kink temperature $T_{\tau}$ in the in-plane torque magnetometry.

For the in-plane torque magnetometry, the magnetic field $\mathbf{H}$ was precisely applied in the $a b$ plane with an error of less than $0.1^{\circ}$. As shown in Fig. 1c, the in-plane response of the magnetic torque exhibits two-fold oscillations with respect to the azimuthal angle $\phi$. We note that the oscillations are dominated only by the two-fold component and the higher-order components due to nonlinear susceptibilities are negligibly small in the whole temperature range we measured. This is evident by the Fourier analysis of the torque oscillations, in which the raw torque curve $\tau(\phi)$ is decomposed as $\tau=\tau_{2 \phi}+\tau_{4 \phi}+\cdots$, where

$\tau_{2 n \phi}=A_{2 n \phi} \sin 2 n\left(\phi-\phi_{0}\right)$ is a term with $2 n$-fold symmetry with $n=1,2, \ldots$ In Supplementary Fig. 3, we plot the amplitudes of the two-, four-, six- and eight-fold oscillations for underdoped YBCO with $p \approx 0.13$. The amplitudes of the nonlinear susceptibilities $(n \geq 2)$ are two to three orders of magnitude smaller than the two-fold $(n=1)$ component, which comes from the anisotropy of linear susceptibility.

In-plane anisotropy of susceptibility. As the average of in-plane susceptibilities $\chi_{\|}=(1 / 2)\left(\chi_{a a}+\chi_{b b}\right)$ is dependent on temperature, we introduce a dimensionless order parameter $\eta \equiv\left(\chi_{a a}-\chi_{b b}\right) /\left(\chi_{a a}+\chi_{b b}\right)$, a diagonal component of a nematic traceless symmetric tensor, to discuss the nematicity properly. Here we refer to the magnetic susceptibility data of the powder samples of $\mathrm{YBCO}^{48}$ to obtain the systematic doping dependence of the denominator. Fairly generally, the magnetic susceptibility of powder samples is given by $\chi_{\text {pow }}=(1 / 3) \chi_{a a}+(1 / 3) \chi_{b b}+(1 / 3) \chi_{c c}$ when off-diagonal terms in the susceptibility tensor can be ignored. By combining this with $\Delta \chi_{\perp}=\chi_{c c}-\chi_{\|}$determined from the out-of-plane torque magnetometry and $\Delta \chi_{\|}=\chi_{a a}-\chi_{b b}$ from the in-plane torque magnetometry, a systematic doping dependence of $\chi_{a a}$ and $\chi_{b b}$ is obtained. Supplementary Fig. 4 depicts the temperature dependence of $\chi_{a a}$ and $\chi_{b b}$ obtained for YBCO with different hole concentrations. For all the doping levels, $\chi_{a a}$ and $\chi_{b b}$ both decrease monotonically as the temperature is lowered. The overall magnitude of $\chi_{a a}$ and $\chi_{b b}$ is also enhanced with doping. It should be noted that the difference between $\chi_{a a}$ and $\chi_{b b}$ exhibits a systematic increase as the holes are doped. This difference actually corresponds to the results of the in-plane torque magnetometry as demonstrated in Fig. 2a-c. Whereas the anisotropy of the in-plane susceptibility $\Delta \chi_{\|}$is dependent on temperature above $T_{\tau}$, when normalized to the form $\eta$ it becomes independent of temperature within the error, indicating that the weak temperature dependence of $\Delta \chi_{\|}$above $T_{\tau}$ is caused by the uniform susceptibility.

In Supplementary Fig. $5 \mathrm{a}-\mathrm{d}$, the temperature dependence of $\Delta \chi_{\|}$for four samples measured here $(p \approx 0.1,0.11,0.13$ and 0.15$)$ is shown over a full temperature range from $T_{\mathrm{c}}$ up to $\sim 290 \mathrm{~K}$, which is limited by our experimental set-up using a variable temperature insert in our superconducting magnet. In the superconducting state below $T_{\mathfrak{c}}$, the torque curve shows hysteresis due to the pinning effect of vortices. In the normal state close to $T_{c}$, the effect of superconducting fluctuations can be seen, which distorts $\tau(\phi)$ from the sinusoidal curve of $\sin 2 \phi$ because of the in-plane anisotropy of the upper critical field. This is demonstrated in Supplementary Fig. 6 by the amplitude of higher-order oscillation $A_{4 \phi}$, which becomes significant below doping-dependent temperatures $T_{\mathrm{sf}}$ (open symbols). Above $T_{\text {sf }}$ we have a systematic evolution of $\Delta \chi_{\|}$with doping: at high temperatures above $T^{*}$ the strongly doping-dependent background component shows a weak temperature dependence with a positive $\mathrm{d} \Delta \chi_{\|} / \mathrm{d} T$, and the kink anomaly is only found at $T_{\tau}$, below which all the data show an increasing trend with decreasing temperature. We find no significant anomaly at the CDW transition temperatures.

Data availability. The data that support the plots within this paper and other findings of this study are available from the corresponding author upon reasonable request.

\section{References}

35. Naito, T., Nishizaki, T., Watanabe, Y. \& Kobayashi, N. in Advances in Superconductivity IX (eds Nakajima, S. \& Murakami, M.) 601-604 (Springer, 1997).

36. Nishizaki, T., Takahashi, Y. \& Kobayashi, N. Phase diagram of interlayer Josephson vortices in underdoped $\mathrm{YBa}_{2} \mathrm{Cu}_{3} \mathrm{O}_{y}$. Physica C 468, 664-668 (2008).

37. Liang, R., Bonn, D. A. \& Hardy, W. N. Evaluation of $\mathrm{CuO}_{2}$ plane hole doping in $\mathrm{YBa}_{2} \mathrm{Cu}_{3} \mathrm{O}_{6+x}$ single crystals. Phys. Rev. B 73, 180505(R) (2006).

38. Hinkov, V. et al. Two-dimensional geometry of spin excitations in the high-transition-temperature superconductor $\mathrm{YBa}_{2} \mathrm{Cu}_{3} \mathrm{O}_{6+x}$. Nature 430, 650-653 (2004).

39. Nishizaki, T. \& Kobayashi, N. Vortex-matter phase diagram as a function of oxygen deficiency in untwinned in $\mathrm{YBa}_{2} \mathrm{Cu}_{3} \mathrm{O}_{y}$. J. Low Temp. Phys. 117, 1375-1379 (1999).

40. Nishizaki, T. \& Kobayashi, N. Vortex-matter phase diagram in $\mathrm{YBa}_{2} \mathrm{Cu}_{3} \mathrm{O}_{y}$. Supercond. Sci. Technol. 13, 1-11 (2000).

41. Nishizaki, T., Shibata, K., Sasaki, T. \& Kobayashi, N. New equilibrium phase diagram of $\mathrm{YBa}_{2} \mathrm{Cu}_{3} \mathrm{O}_{y}$ under high magnetic fields. Physica C 341-348, 957-960 (2000).

42. Okazaki, R. et al. Rotational symmetry breaking in the hidden-order phase of $\mathrm{URu}_{2} \mathrm{Si}_{2}$. Science 331, 439-442 (2011).

43. Kasahara, S. et al. Electronic nematicity above the structural and superconducting transition in $\mathrm{BaFe}_{2}\left(\mathrm{As}_{1-x} \mathrm{P}_{x}\right)_{2}$. Nature 486, 382-385 (2012).

44. Watanabe, D. et al. Novel Pauli-paramagnetic quantum phase in a Mott insulator. Nat. Commun. 3, 1090 (2012).

45. Kasahara, S. et al. Giant superconducting fluctuations in the compensated semimetal FeSe at the BCS-BEC crossover. Nat. Commun. 7, 12146 (2016).

46. Watanabe, T., Fujii, T. \& Matsuda, A. Pseudogap in $\mathrm{Bi}_{2} \mathrm{Sr}_{2} \mathrm{CaCu}_{2} \mathrm{O}_{8+\delta}$ studied by measuring anisotropic susceptibilities and out-of-plane transport. Phys. Rev. Lett. 84, 5848-5851 (2000).

47. Wang, Y. et al. Field-enhanced diamagnetism in the pseudogap state of the cuprate $\mathrm{Bi}_{2} \mathrm{Sr}_{2} \mathrm{CaCu}_{2} \mathrm{O}_{8}$ superconductor in an intense magnetic field. Phys. Rev. Lett. 95, 247002 (2005).

48. Leridon, B., Monod, P., Colson, D. \& Forget, A. Thermodynamic signature of a phase transition in the pseudogap phase of $\mathrm{YBa}_{2} \mathrm{Cu}_{3} \mathrm{O}_{x}$ high- $T_{c}$ superconductor. Euro. Phys. Lett. 87, 17011 (2009). 